\title{
Opportunities for Microtechnology In Metrology
}

Davey Wijngaards and
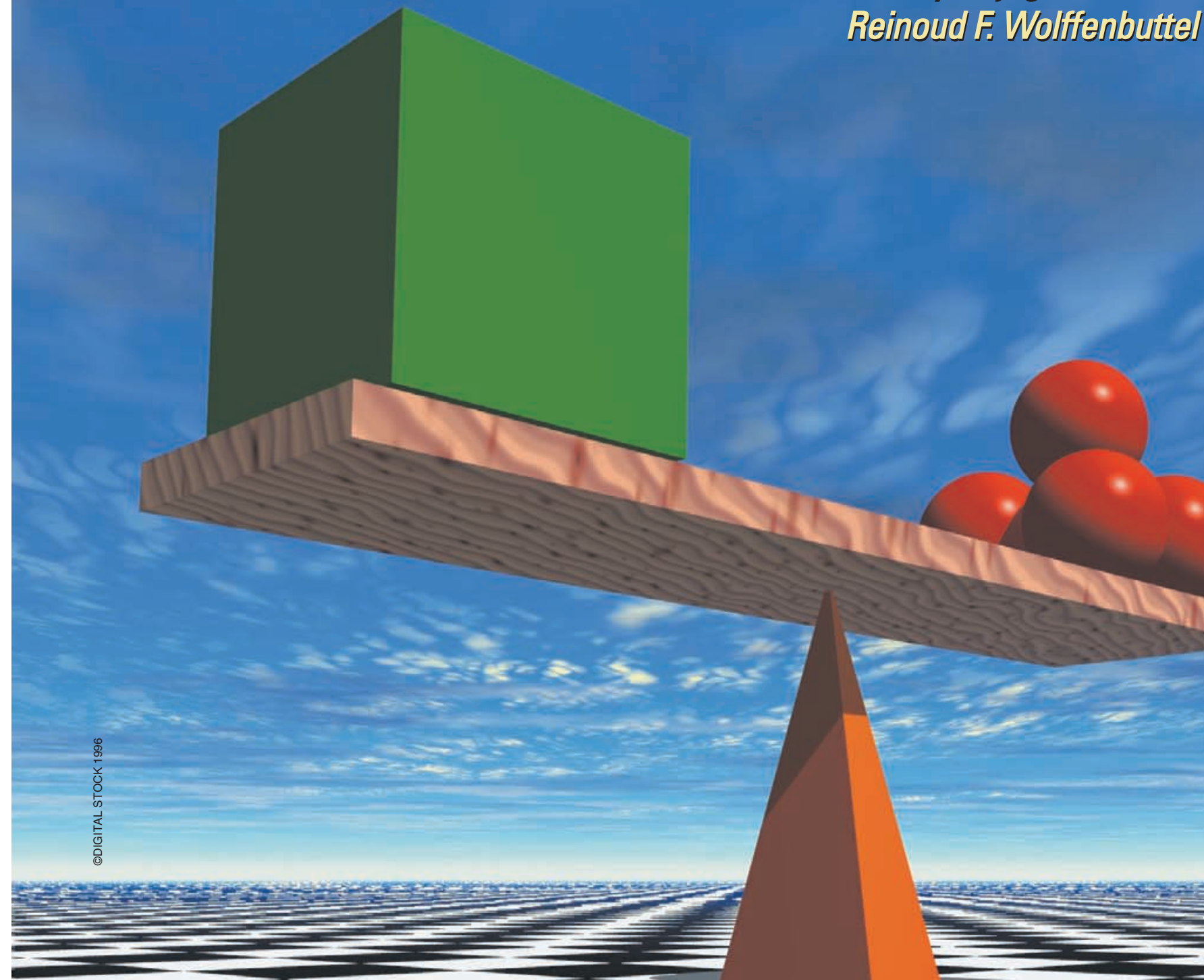

Remoud $F$ Wholffenbutter
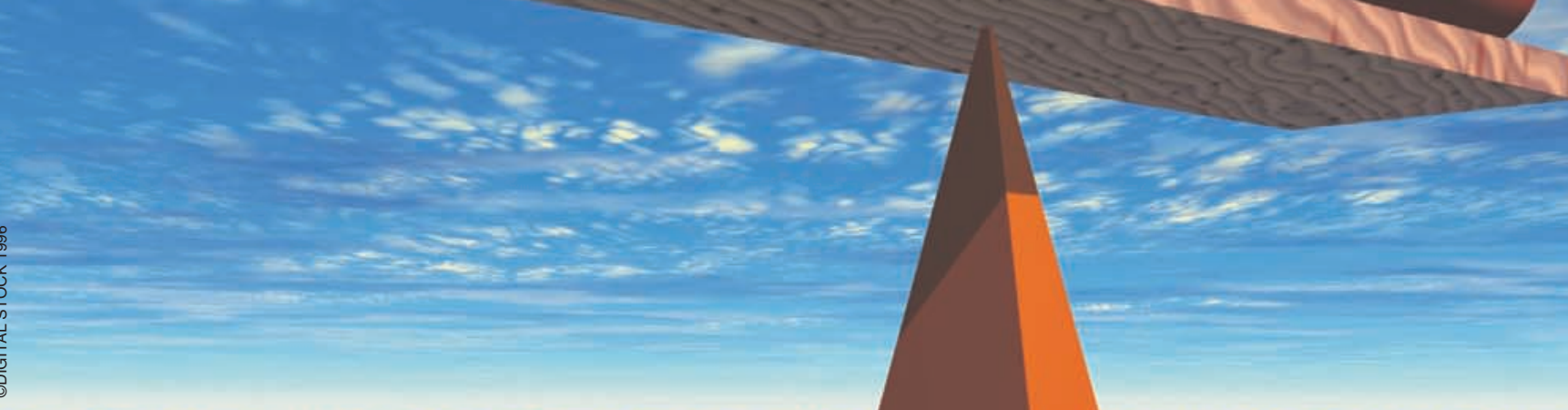
ilicon sensor technology originated from the mainstream fabrication of integrated circuits. From there, it evolved not only into a technology for high-volume batch fabrication of silicon sensors, but also into technologies for the fabrication of micromechanical structures. One of the main drivers for these developments has been material compatibility, which allows the integration of sensors and circuits in the silicon substrate, enabling the fabrication of microsystems on the smallest possible carrier. In practice, introduction of fully integrated microsystems has proven more difficult than anticipated.

What is the reason for this apparent gap between the availability of sensor technology and the application of that same technology to integrated microsystems? The availability of high-quality wafers and equipment is undisputed, but the introduction of batch fabrication techniques in silicon sensor manufacturing has, in only a few cases, lead to high-volume production of on-chip integrated silicon microsystems. The diversity of components in a microsystem and the need to tailor these components for each specific application have prevented high-volume production. Still, the concept has been demonstrated and proven crucial in specific applications such as the metrological applications discussed in this article.

\section{Microtechnological Systems}

One of the virtues of microtechnology is the option to integrate the electronics and transducers on a single die. This allows the existing characteristics of a device to be enhanced, for example, by adding signal conditioning or self-test and self-calibration facilities. A second advantage is the availability of a large infrastructure from the microelectronic and microtechnological processing industry. Third, the high quality of the base materials and processing equipment provides highly reproducible devices.

\section{Hybrid Versus Integrated Systems}

Even though the number of new devices, technologies, and materials is staggering, only three types of system implementations are distinguished: a hybrid, semihybrid, or fully integrated system (listed in order of increasing level of complexity). In the hybrid and semihybrid solution, the electronics and the transducers are fabricated separately and merged afterwards, using a passive carrier in the case of a hybrid system, and an active carrier or wafer bonding in the case of a semihybrid system. A (semi)hybrid system has the primary advantages of "known good die" selection prior to merging, which increases yield [8], and reduces cost, and allows more relaxed fabrication constraints. This allows for the use of materials and fabrication techniques that are otherwise incompatible with standard integrated circuit (IC) fabrication techniques.

So, if a hybrid system is so flexible and effective, why do we need integration? The answer is that in many cases we don't, but in some cases we cannot do without it. We need integration:

Do minimize the number of components (e.g., a single-chip solution),
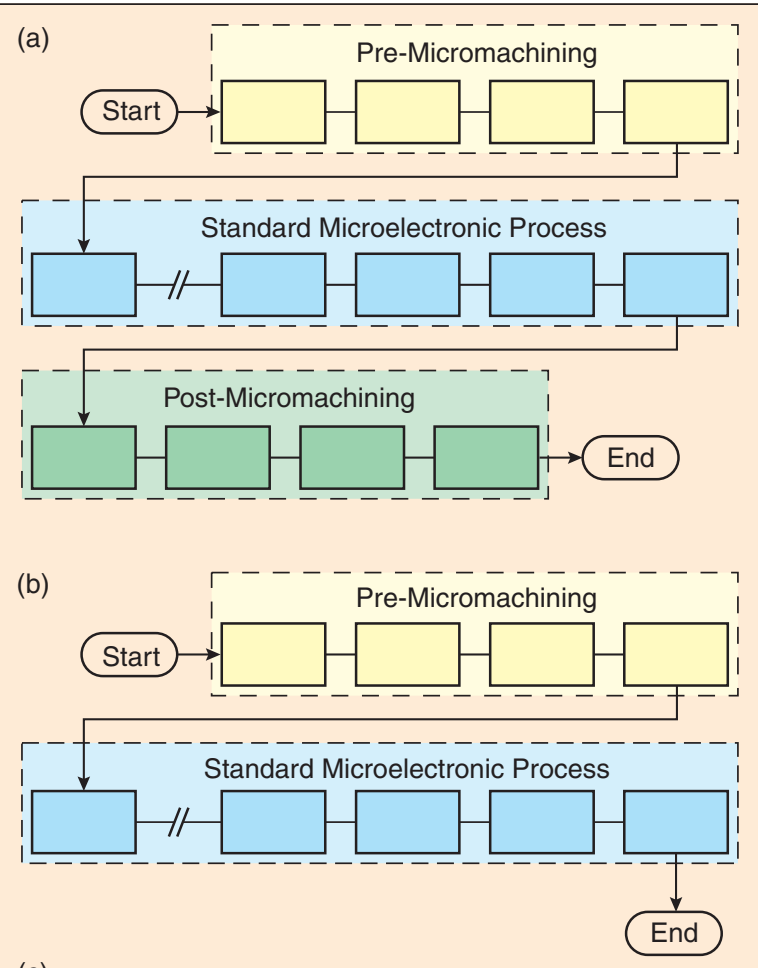

(c)

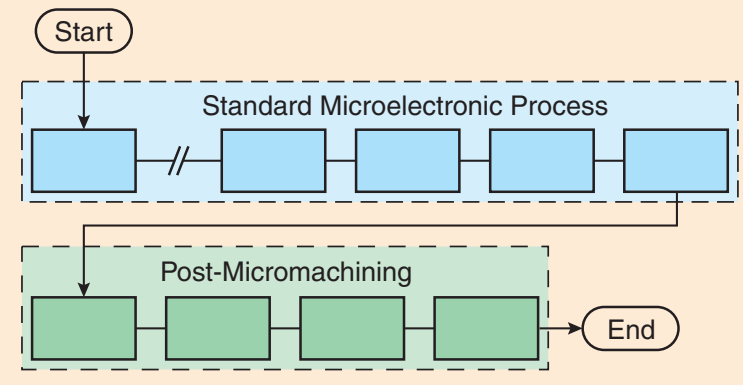

(d)

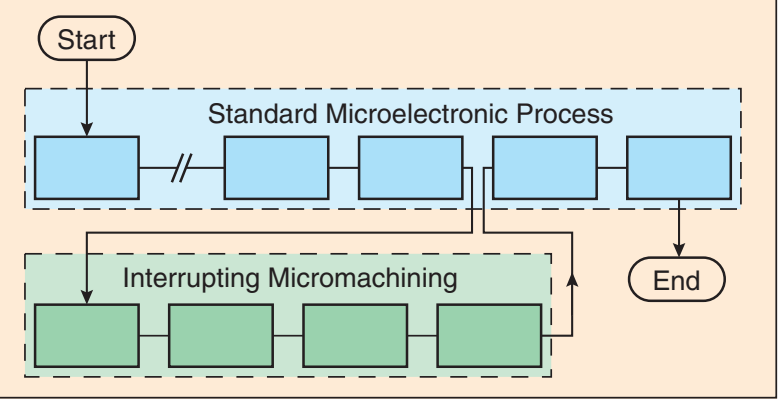

Fig. 1. Modular on-chip integration strategies, incorporating (a) pre- and post-processing, (b) pre-processing only, (c) post-processing only, and (d) interrupting micromachining.

D to reduce volume (e.g., to fit a biomedical sensor into a catheter),

to have on-chip self-test and self-calibration facilities available,

D to reduce the susceptibility to environmental noise [1] and increase overall reliability, by reducing the length and the number of the leads between the sensor and/or actuator part and the circuits. 


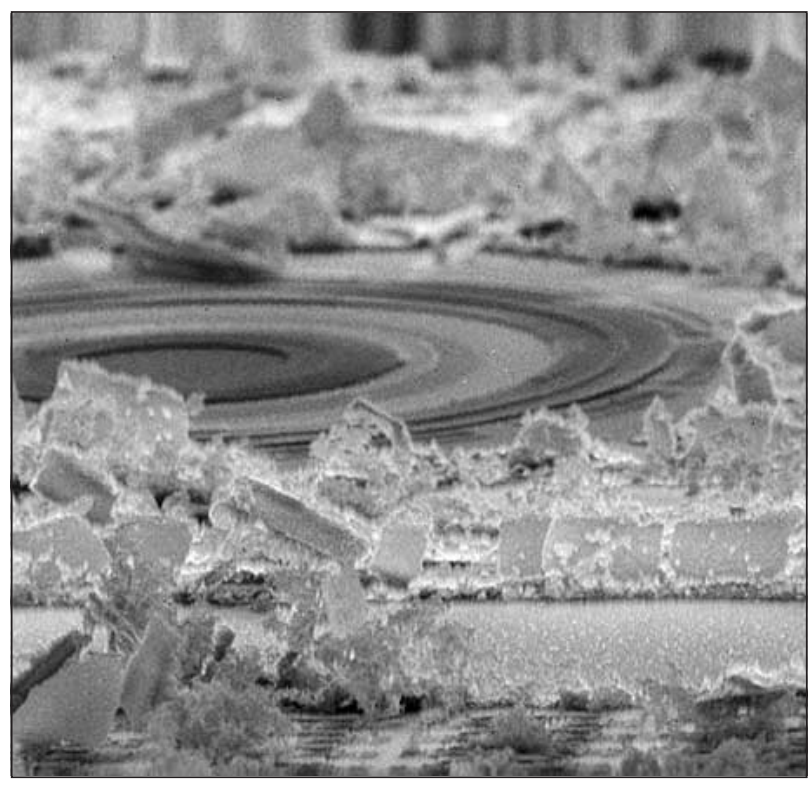

Fig. 2. Aluminum attack during sacrificial etching, rendering a micromotor useless. (Courtesy of J.F.L. Goosen, Delft University of Technology, The Netherlands.)

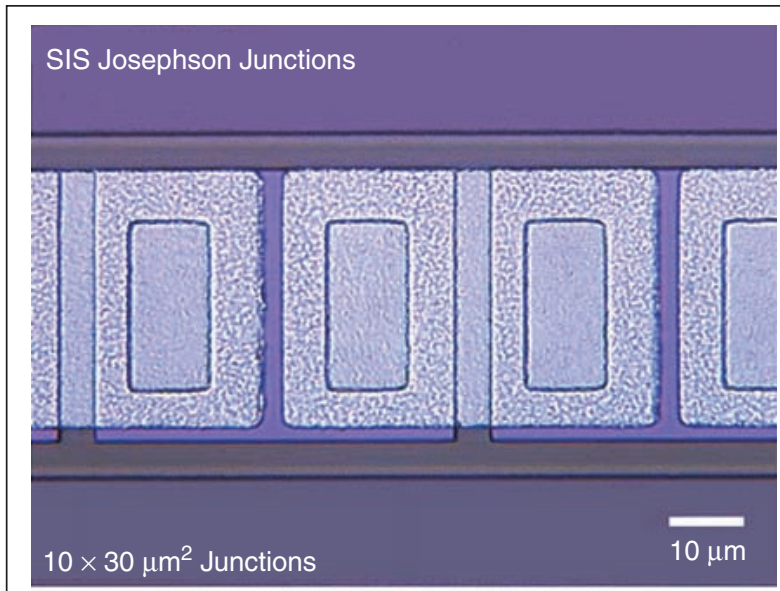

SNS Josephson Junctions

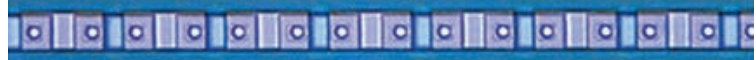

$2 \times 2 \mu \mathrm{m}^{2}$ Junctions

Fig. 3. Comparison of the 20-year-old conventional Nb- $\mathrm{Al}_{2} \mathrm{O}_{3}-\mathrm{PblnAu} 18 \times 30$ $\mu \mathrm{m}^{2}$ SIS junctions with the Nb-PdAu-Nb $2 \times 2 \mu \mathrm{m}^{2}$ SNS junctions. The contact holes of the SNS JJS is only $1 \times 1 \mu \mathrm{m}^{2}$. SNS arrays are distributed along the center conductor of a coplanar waveguide. (Courtesy: S.P. Benz, NIST, USA.)
One way to achieve integration is through a fully customized process. Unfortunately, customization is inflexible, hardly allowing any changes in the fabrication process. Moreover, customization requires a lot of dedicated equipment because of issues with clean room re-entrance. This makes a custom process very expensive, both in startup costs and infrastructure. So, unless high-volume production is anticipated, the usefulness of a fully customized process is limited. In contrast, modular fabrication-the consecutive processing of the transducer and electronics - can circumvent these problems [9]. Fig. 1 shows the most common paths. Practically, post-processing of the transducer, as depicted in Fig. 1(c), is the preferred technique. However, an interruption late in the microelectronic process, as shown in Fig. 1(d), may be unavoidable at times. A common example is to deposit the aluminum (Al) metallization (as part of the electronic interconnect) after bulk micromachining has taken place, as the $\mathrm{Al}$ would otherwise be attacked. Fig. 2 shows exactly how disastrous such an attack can be.

\section{Fabrication Compatibility}

To assure the successful fabrication of an integrated device, the device needs to be fabrication compatible, which encompasses three issues:

D Material compatibility-All materials must be compatible with each other and survive processing.

D Process compatibility-Micromachining of the transducer must not interfere with the performance of the electronics and vice versa. Interference can appear due to 1) temperature steps, referred to as an increment in thermal budget, that degrade the performance of either the electronics or the transducer, and 2) the attack on a material during one of the processing steps (e.g., attack of the aluminum interconnect during bulk micromachining).

D Equipment contamination restrictions-Clean room re-entrance is restricted after transducer processing (e.g., wafers are generally not allowed back into a standard microelectronic processing line after etching in an alkaline solution).

Modular fabrication techniques have a twofold advantage. First, the interchangeability of the modules is optimized; each module has to be developed only once and can be re-used in another process. Second, as the transducer fabrication module doesn't interfere with the microelectronic process, the existing microelectronic infrastructure can be used without a lot of restrictions. Today, many fabrication-compatible micromachining techniques are available and can be divided into three categories: bulk micromachining, surface micromachining, and thin-film fabrication techniques. An exhaustive overview of these three processing techniques goes beyond the scope of this article, but a few interesting examples are reviewed.

\section{System Cost}

Regardless of the technology chosen, the total cost of a system remains a controversial issue, as testing and packaging, rather than the fabrication of the device itself, are responsible for most of the cost. Consequently, the self-test capabilities of an 
integrated system could even help reduce testing costs prior to packaging. Using the airbag sensor as an example, Ford chose a hybrid solution, comprising a separate sensor die and a readout chip that are wire bonded and glued in a single package [10]. ICS and Lucas/Novasensor, on the other hand, thought it was better to go for a wafer-bonded solution [2]. Analog Devices even went a step further and developed an accelerometer that was fully integrated with signal conditioning and readout electronics [4].

\section{Examples from Metrology}

Microtechnology has helped develop some very successful metrological devices. Two particular devices: the Josephson Junction Array (JJA) [3] and the Multi-Junction Thermal Converter (MJTC) [6] are detailed below.

\section{Josephson Junction Array}

This dc voltage reference standard is based on the Josephson effect that directly relates voltage to the frequency of an actuating microwave signal, through a ratio of fundamental constants. Since frequency can be accurately and stably controlled to parts in $10^{12}$, a Josephson junction can accurately convert a frequency into voltage. The device consists of a large number of junctions in series along the center of a planar waveguide that couples the microwave signal into the Josephson array. Because the Josephson effect is a superconducting effect, the array has to operate at cryogenic temperatures $(<4.2 \mathrm{~K})$. This requires a measurement setup around a liquid-helium cooled dewar with special equipment to bring the microwave down to the array in the dewar and bring the dc signal from the array

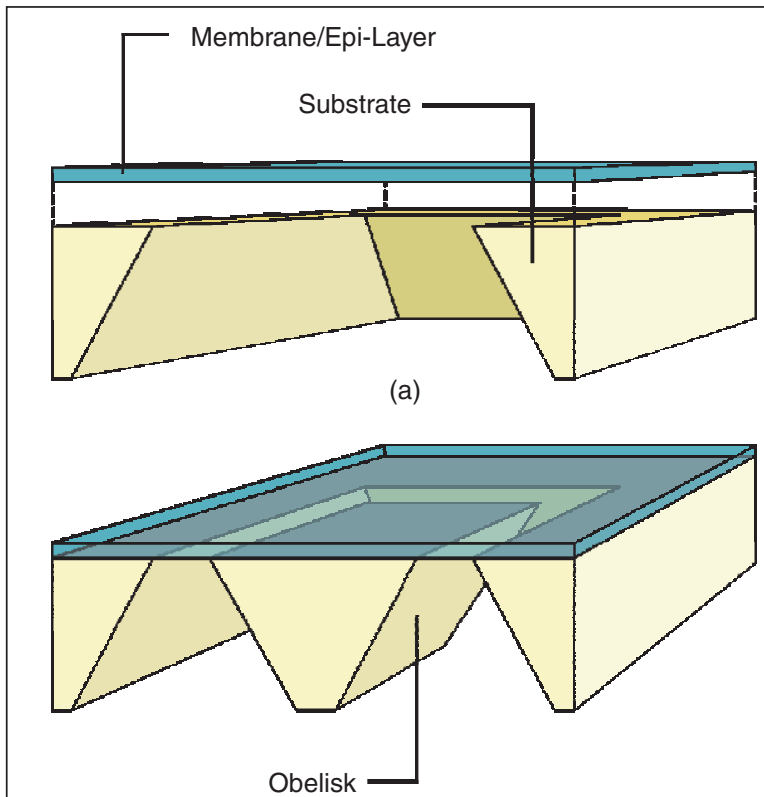

(b)

Fig. 4. Cross-sectional view of the bulk micromachined substrate (a) without and (b) with an obelisk present. The obelisk is used to change the thermal time constant of the device from $25 \mathrm{~ms}$ to $1.3 \mathrm{~s}$, which is required for $\mathrm{AC}$ reference to operate near $10 \mathrm{~Hz}$. back out. This arrangement limits the portability of such a reference standard.

The initial JJA was a single junction, capable of generating no more than a few millivolts. Improvements in microfabrication technology reduced the junction size drastically, while greatly increasing the number of junctions. This has lead to arrays of 20,000 junctions with a nominal output voltage between $1 \mathrm{~V}$ and $10 \mathrm{~V}$, as shown in Fig. 3. The next step is the realization of programmable reference standards based on Josephson arrays (referred to as the Josephson arbitrary waveform generator). This basic idea will help realize selectable dc voltages and, furthermore, a fundamental ac voltage standard. However, to create a lumped array capable of delivering a $1 \mathrm{~V}$ output requires 13,500 junctions in series, spaced only $120 \mathrm{~nm}$ apart. This is a significant challenge, since the smallest arrays still have a junction spacing of $7 \mu \mathrm{m}$.

\section{Multi-Junction Thermal Converter (MJTC)}

The MJTC is a thermal RMS-to-dc converter. Operation is based on the Joule heating of a heating resistor driven by ac current, which causes a rise in the steady-state temperature of the thermal mass. Thermocouples detect the difference in temperature between ambient and the device. Fig. 4 shows the schematic of the device, with and without the use of an obelisk to improve the low-frequency response of the device. The actual device is shown in Fig. 5. The entire device can be constructed using only fabrication-compatible technology:

D Fabrication of the stress-compensated $\mathrm{Si}_{3} \mathrm{~N}_{4} / \mathrm{SiO}_{2} / \mathrm{Si}_{3} \mathrm{~N}_{4}$ membrane-These dielectric materials are standard processing materials.

D Deposition of the thin film metals-A generic thin-metal film deposition technique can deposit both the $\mathrm{NiCr}$ heater and the $\mathrm{Cu}-\mathrm{Cu}_{0.56} \mathrm{Ni}_{0.44}$ thermocouples [11].

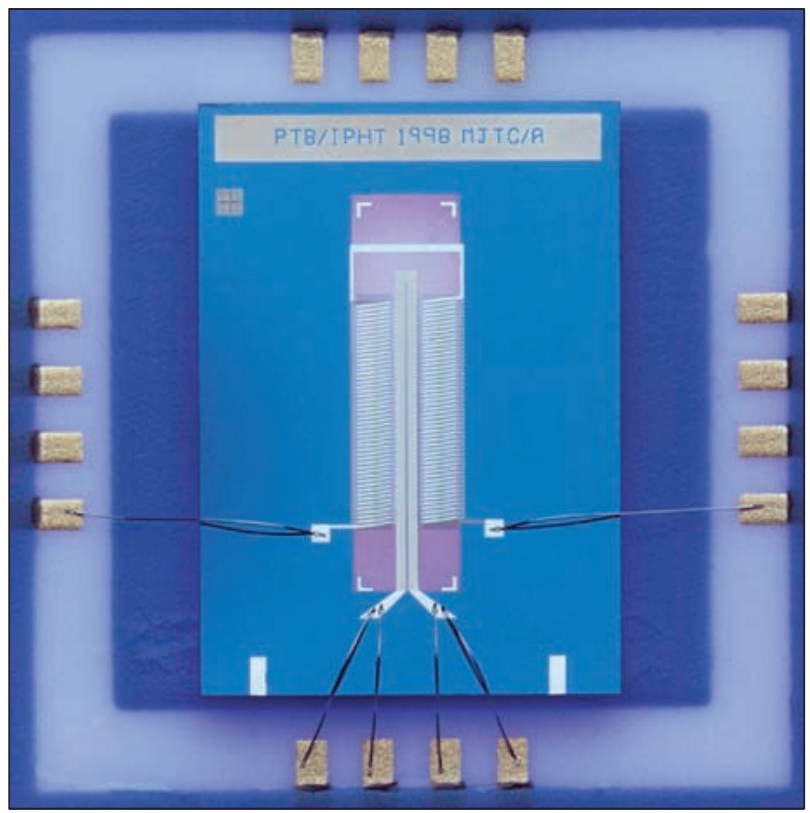

Fig. 5. Photo of the Planar MJTC chip on its $\mathrm{Al}_{2} \mathrm{O}_{3}$-carrier. Al-bond wires connect the Al pads on the chip to the Ag pads on the carrier. (Courtesy M. Klonz, PTB, Germany.) 


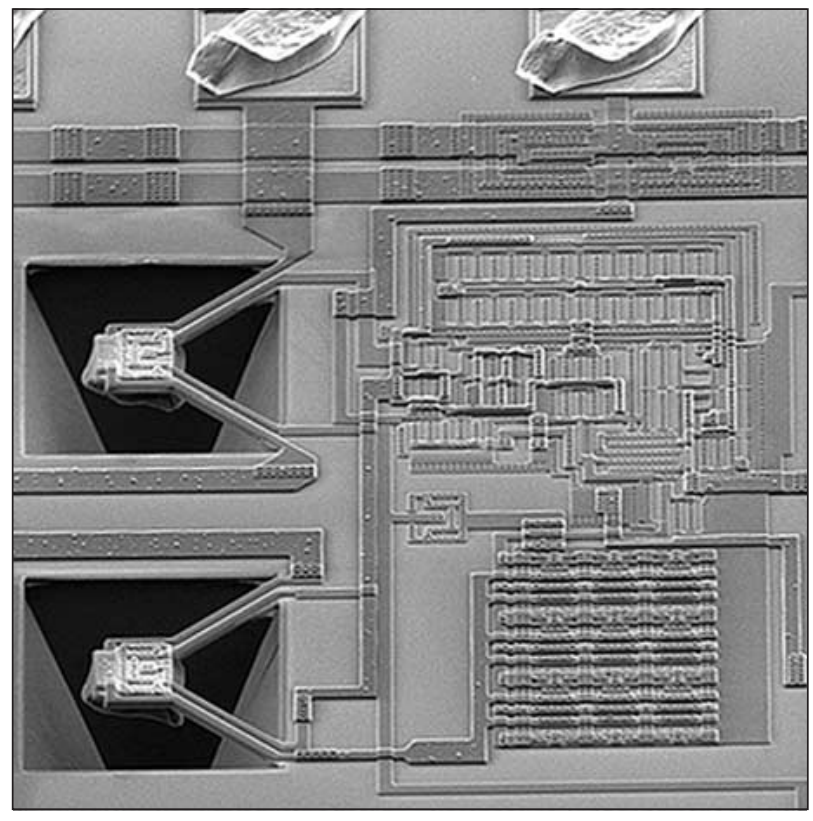

Fig. 6. A scanning electron micrograph of an RMS converter as developed by Klaassen et al. The converters (on the left) are thermally isolated by bulk micromachining.

D Bulk micromachining of the substrate-Anisotropic etching can selectively remove the substrate, using an electrochemical etch stop to conserve the obelisk.

\section{The Opportunies}

To our knowledge, the JJA and the thermal RMS-to-dc converter are the only commercially available metrological applications that have employed microtechnology. From these two examples, three specific features of microtechnology stand out:

D The technological advances in lithography allow device geometries to shrink continually. The main drawback is that the thermal budget scales down with feature size.

- Improvements in processing exotic material, especially thin-film deposition techniques, have made improved materials available.

- Reliable bulk and surface micromachining facilities can sculpt the substrate as required.

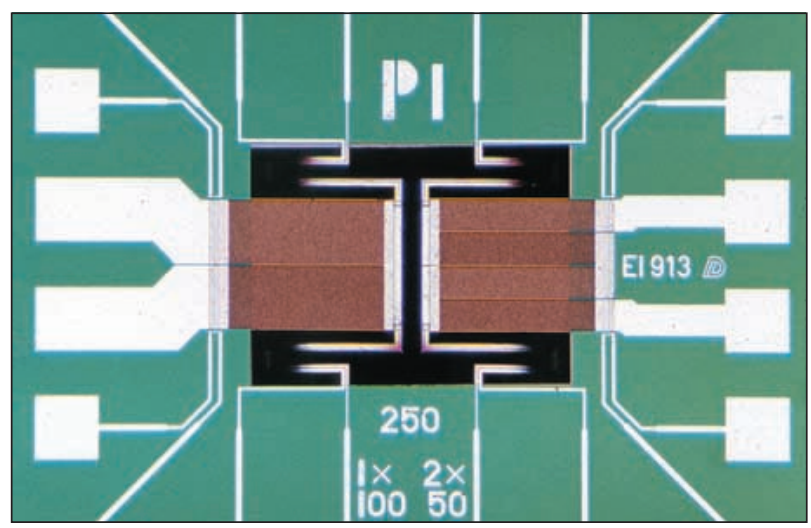

Fig. 8. Photograph of a single and double pair of Peltier elements, with respective thermoelement dimensions of $250 \times 100 \mu \mathrm{m}$ and $250 \times 50 \mu \mathrm{m}$

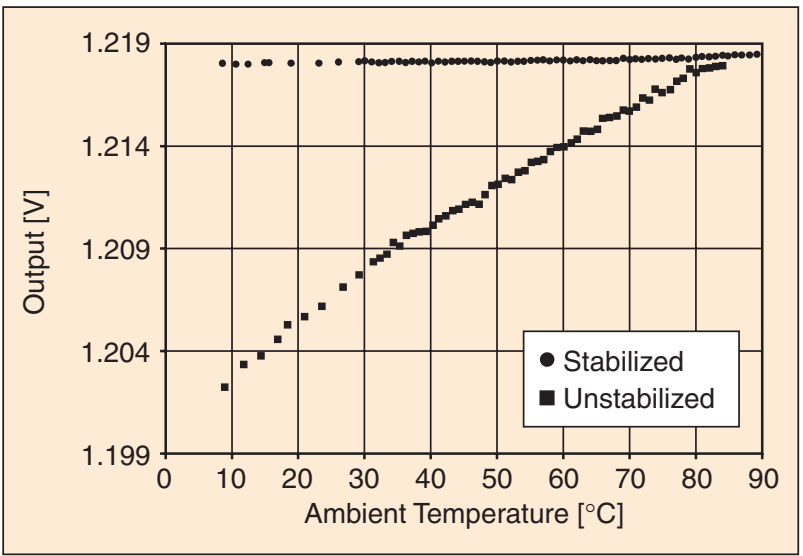

Fig. 7. The output of a thermally isolated bandgap reference, with and without stabilization by active heating. (Courtesy E.H. Klaassen, Stanford University, USA.)

\section{Applications for the Near Future}

The successful use of microtechnology is not limited to the two applications above. Developments in electrostatic RMS-to-dc converters, as an alternative ac standard, and single electron tunneling devices, for electrical current standards, depend heavily on the latest advances in microelectromechanical systems (MEMS). Three specific types of instrumentation and measurement applications can benefit from a closer collaboration between metrology and microtechnology:

Improved or new metrological references,

D Portable standards,

Dn-chip references.

Where the next steps in the development of new references will come from remains to be seen. In the case of the JJA, the main drive came from metrology. In the case of the single electron tunneling device, and the thermal and electrostatic RMS-to-dc converters, developments are growing in collaboration with microtechnology. The option of integrating references and electronics on a single die should make it possible to significantly reduce the size of the existing reference systems. This should allow for the development of portable standards that play an increasingly important roll for calibrations in the field where equipment downtime is important.

The development of on-chip references is less of a metrological challenge, as these will primarily deal with keeping less than ideal sensors within specification. However, applications such as a microinstrumentation platform have a much larger functionality than regular sensors by merging multiple sensors on a single active die. The proper operation of each component requires self-test and self-calibration facilities, along with an on-chip reference for the self-calibration. This requires additional, or improved, functionality through the use of new materials, new technological solutions, or improvements in microelectronics.

A good example of such an improvement is the development of bandgap references. Basically, the bandgap voltage is extracted from the base-emitter voltage of a pair of bipolar substrate transistors [7]. One of the problems with these de- 
vices is temperature dependency. A good technique, presented by Klaassen et al. [5], is the use of active heating and passive cooling of a bandgap reference that is located on a thermally isolated island. Fig. 6 shows the device. Fabrication-compatible, front-side micromachining created the thermal isolation. First, the oxide and other dielectric layers were patterned, then the device was exposed to an etchant which anisotropically removed the substrate through the areas where the dielectric layers were removed. An electrochemical etch stop preserves the epi-layer, in which the circuit is located, to preserve the bandgap circuit. Fig. 7 compares the significant improvement in stability of the thermally stabilized bandgap voltage to an unstabilized bandgap voltage. As the active heating technique requires the reference to be heated well above the ambient temperature, the next logical step is to employ active, rather than passive cooling, to reduce the operating temperature. One possible way to do this is to use on-chip integrated Peltier elements similar to the device shown in Fig. 8 [12].

\section{Summary}

The vast infrastructure of the microelectronic and microtechnological processing industry has yielded highly reproducible devices through reliable, reproducible, and fabrication-compatible processing techniques. Much development has occurred in bulk and surface micromachining, as well as in thin-film deposition techniques. Nevertheless, only a limited number of metrological applications have benefited from the use of this technology.

Only two metrological applications that use microtechnology have been developed into commercially available devices: the JJA, as a dc reference, and the thermal RMS-to-dc converter, as an ac reference. Single electron tunneling devices and micromachined electrostatic RMS-to-dc converters are still under development. The accuracy requirements of the existing applications will continue to increase. This makes the development of on-chip references and on-chip self-test and self-calibration facilities essential. These microelectronic features, until now, have remained unexploited in metrology, as well as in most other applications.

\section{References}

[1] A. Bakker and J.H. Huijsing, "Low-offset low-noise 3.5mW CMOS spinning-current Hall effect sensor with integrated chopper amplifier," in Proc. Eurosensors XIII, The Hague, The Netherlands, Sept. 12-15, 1999, pp. 1045-1048.

[2] P.W. Barth, "Silicon fusion bonding for fabrication of sensors, actuators and microstructures," Sens. Actuators A, Phys., vols. 21-23, pp. 919-926, 1990.

[3] S.P. Benz, P.D. Dresselhaus, and C.J. Burroughs, “Nanotechnology for next-generation Josephson voltage standards," in Proc. Symp. on Microtechnology in Metrology and Metrology in Microsytems, Delft, The Netherlands, Aug. 31-Sept. 1, 2000, pp. 7-30.

[4] K.H.-L. Chau, R.S. Lewis, Y. Zhao, S.F. Bart, and R.G. Marcheselli, "An integrated force-balance capacitive accelerometer for low-g application," in Proc. Transducers '95, Stockholm, Sweden, June 25-29, 1995, vol. 1, pp. 593-596.

[5] E.H. Klaassen, R.J. Reay, and G.T.A. Kovacs, "Diode-based thermal RMS converter with on-chip circuitry fabricated using CMOS technology," Sens. Actuators A, Phys., vol. 52, pp. 33-40, 1996.

[6] M. Klonz, H. Laiz, and E. Kessler, “Development of thin-film multijunction thermal converters in PTB/IPHT," in Proc. Symposium on Microtechnology in Metrology and Metrology in Microsytems, Delft, The Netherlands, Aug. 31-Sept. 1, 2000, pp. 7-30.

[7] G.C.M. Meijer, G. Wang, and F. Fruett, "Integrated voltage references and temperature sensors in CMOS technology," in Proc. Symp. on Microtechnology in Metrology and Metrology in Microsytems, Aug. 31-Sept. 1, 2000, Delft, The Netherlands, pp. 69-78.

[8] T.L. Michalka, R.C. Varchney, and J.D. Meindl, “A discussion on yield modelling with defect clustering, circuit repair and circuit redundancy," IEEE Trans. Semiconduct. Manufact., vol. 3, pp. 116-127, Mar. 1990.

[9] P.M. Sarro, "Sensor technology strategy in silicon," Sens. Actuators A, Phys., vol. 31, pp. 138-143, 1990.

[10] L. Spangler and C.J. Kemp, "Integrated silicon automotive accelerometer," in Proc. Transducers '95, Stockholm, Sweden, June 25-29, 1995, vol. 1, pp. 585-588.

[11] D.D.L. Wijngaards, M. Bartek, and R.F. Wolffenbuttel, "Silicon IC process compatible thin metal film post-processing module," Sens. Actuators A, Phys., vol. 68, pp. 419-428, 1998.

[12] D.D.L. Wijngaards, S.H. Kong, M. Bartek, and R.F. Wolffenbuttel, "Design and fabrication of on-chip integrated polySiGe and polySi Peltier devices," in Sens. Actuators A, Phys., vol. 85, pp. 316-324, 2000

Davey Wijngaards received an M.Sc. degree in electrical engineering, cum laude, from the Delft University of Technology in 1998. He is currently a Ph.D. candidate with the Electronic Instrumentation Laboratory at the Delft University of Technology. As part of the STW project (Microinstrumentation Platform in Silicon), he is performing research on thermal stabilization using on-chip intregrated Peltier devices.

Reinoud F. Wolffenbuttel received an M.Sc. degree in 1984 and a Ph.D. degree in 1988, both from the Delft University of Technology. He is an Associate Professor at the Laboratory of Electronic Instrumentation at the Delft University of Technology and is involved in instrumentation and measurement in general and on-chip functional integration of microelectronic circuits, silicon sensor fabrication compatibility issues, and micromachining in silicon and microsystems in particular. 